\title{
CULTIVOS TRANSGÉNICOS EN AMÉRICA LATINA: expropiación, valor negativo y Estado
}

\author{
Pablo Lapegna* \\ Gerardo Otero**
}

Resumen: ¿Cuál es la relación entre el régimen alimentario neoliberal y los cultivos transgénicos en la agricultura de América Latina? En este artículo nos proponemos dos objetivos. En primer lugar, buscamos proporcionar las principales definiciones conceptuales y los parámetros analíticos que contextualizan los estudios de caso compilados en este número especial derivados del Simposio de Cultivos Transgénicos y Neoliberalismo en América Latina. En segundo lugar, utilizamos dichos textos como la base empírica para elaborar brevemente los conceptos de expropiación, acumulación por desposesión y valor negativo, los cuales comprenden las consecuencias más trascendentes del régimen alimentario neoliberal. También ofrecemos una breve descripción de los artículos, vinculándolos a nuestra propuesta teórica. Esperamos que este simposio estimule la investigación sobre los vínculos entre cultivos transgénicos y las dinámicas del desarrollo capitalista mundial.

Palabras clave: neoliberalismo, biotecnología, agricultura, régimen alimentario, América Latina.

* Profesor asistente del Departamento de Sociología y el Instituto de Estudios Latinoamericanos y del Caribe (LACSI), Universidad de Georgia, Estados Unidos. Correo-e: plapegna@uga.edu

** Profesor de la Escuela de Estudios Internacionales, Universidad Simon Fraser, Canadá. Correo-e: otero@sfu.ca 


\section{TRANSGENIC CROPS IN LATIN AMERICA: expropriation, negative value and the State}

Abstract: This paper introduces a symposium on transgenic crops and neoliberalism in Latin America. We address the question: What is the relationship between the neoliberal food regime and transgenic crops in Latin American agriculture? Our goals are, first, to provide the main conceptual definitions and analytical parameters to contextualize the case studies that follow; and, second, using the findings of our contributors as our empirical stepping stone, to briefly elaborate the concepts of expropriation, accumulation by dispossession and negative value as the primary consequences of the neoliberal food regime. We also offer a brief description of each of the papers in the symposium that follows, linking them to our theoretical proposal. We hope this symposium will help in further exploration of the connection between GM crops and the larger dynamics of capitalist development worldwide.

Keywords: neoliberalism, biotechnology, agriculture, food regime, Latin America. 


\section{Introducción}

Los cultivos transgénicos o genéticamente modificados (GM) fueron introducidos en América Latina en 1996, cuando el gobierno argentino aprobó la comercialización de soja resistente al herbicida glifosato. A partir de entonces la producción de soja GM (y otros cultivos transgénicos como el maíz y el algodón) se expandió en la región. Esta difusión inició en la década de 2000, época en que la soja transgénica fue adoptada en Uruguay. Las semillas de soja transgénica también fueron contrabandeadas desde Argentina e ilegalmente sembradas en Paraguay y el sur de Brasil a fines de la década de 1990, situación que se utilizó para legalizar los cultivos GM en esos países en 2004 y 2005, respectivamente (Hisano \& Altoé, 2008; Hetherington, 2013). Pocos años después, en 2011, se sembraban cultivos transgénicos en 66 millones de hectáreas en América del Sur (Santiago, 2012), lo que representa 40 por ciento de la superficie mundial plantada con tales cultivos.

El uso de semillas transgénicas ha sido un tema muy controvertido y discutido en México y América Central, donde los cultivos GM son percibidos como una amenaza para la soberanía alimentaria nacional y la biodiversidad. Ello ha dado lugar a una serie de movilizaciones populares, campañas y acciones judiciales que ha establecido moratorias para el uso de semillas genéticamente modificadas (Keplek, 2012). En América del Sur, sin embargo, estos cultivos han sido ampliamente adoptados, a pesar de la fuerte oposición en Brasil. Argentina y Brasil poseen una larga historia de integración a los mercados mundiales de materias primas. En ambos países los sectores latifundistas y los agricultores capitalizados tienen un peso importante en la economía política agraria. Las organizaciones de la sociedad civil de Brasil se opusieron en un inicio a los cultivos transgénicos desde 1996 y lograron una moratoria hasta 2005. El papel de las organizaciones campesinas fue crucial, como el Movimiento 
de los Trabajadores Rurales Sin Tierra (MST), una de las mayores organizaciones campesinas del mundo. Aun así, terratenientes y grupos de presión de Monsanto lograron la legalización de la soja transgénica (Motta, 2016).

Mesoamérica es el principal centro de biodiversidad del maíz, donde se originó hace unos diez mil años (Kloppenburg, 2004). El maíz —al menos, la variedad blanca- se utiliza principalmente como alimento para el consumo humano. De ahí la fuerte oposición al maíz transgénico (aunque este último es la variedad amarilla, que se emplea sobre todo como forraje o alimento para ganado), porque podría «contaminar» con facilidad o comprometer la biodiversidad del maíz (Fitting, 2011). La soja, por el contrario, se produce en Sudamérica como cultivo de forraje, para los mercados de exportación. Cabe mencionar que su producción cambia el uso de la tierra, de la producción de comida a la de forrajes, por lo que amenaza la seguridad alimentaria (Teubal, 2008); fomenta la expulsión de campesinos y pueblos indígenas, además de la deforestación de bosques nativos; $y$ provoca problemas graves de salud pública debido a la exposición a herbicidas (Pengue, 2005; Hetherington, 2013; Lapegna, 2013; Leguizamón, 2014).

La finalidad del simposio sobre transgénicos y neoliberalismo en América Latina es documentar algunas de las primordiales consecuencias sociales y ecológicas de esta convergencia tecnológica y política. El «giro biotecnológico» en la agricultura latinoamericana se inserta en el movimiento global hacia la «carnificación» (meatification) del consumo alimentario (Weis, 2013). Así, el aumento de la producción y el consumo de carnes (res, pollo o puerco), accesibles para las clases medias y de ingresos más altos, se asocia con una mayor desigualdad alimentaria (Otero, Pechlaner, Liberman, Gürcan, 2015). La «carnificación» también ha intensificado diversos problemas ecológicos (Weis, 2013) y de salud (Lang, 2010). Estas pueden ser las razones por las cuales la Organización 
de las Naciones Unidas (ONU) designó a 2016 como el año de las legumbres o leguminosas (frijoles, chícharos, garbanzos y lentejas). Puesto que 75 por ciento de las leguminosas se consumen como fuente fundamental proteica en los países en vías de desarrollo (Hui, 2016), su sustitución podría plantearse como la nueva frontera para los productores de soja y, en última instancia, de los productores de carne. No obstante, los datos en torno de la eficiencia socioeconómica de tal sustitución son contundentes: la producción de un kilogramo de legumbres resulta en 0.5 kilogramos de $\mathrm{CO}_{2}$, en comparación con 9.5 kilogramos de $\mathrm{CO}_{2}$ por un kilogramo de carne vacuna. Las consecuencias de esta «carnificación» podrían ser, en definitiva, nefastas para el cambio climático.

En ese sentido, cuestionamos ¿cuál es la relación entre el régimen alimentario neoliberal y los cultivos transgénicos en la agricultura latinoamericana? Nos proponemos dos objetivos con la intención de responder la pregunta. En primer lugar, buscamos proporcionar las definiciones conceptuales más relevantes y los parámetros analíticos que contextualizan los estudios de casos aquí compilados. En segundo lugar, utilizamos estos artículos como la base empírica para elaborar los conceptos de expropiación, acumulación por desposesión y valor negativo, que son consecuencias del régimen alimentario neoliberal. Ofrecemos también una breve descripción de los artículos, relacionándolos con nuestra propuesta teórica.

\section{Régimen agroalimentario neoliberal}

Los alimentos han tenido un lugar central en la acumulación de capital desde el inicio del capitalismo, como una esfera para la obtención de beneficios en sí misma y de forma central como uno de los componentes básicos de la reproducción de la fuerza de trabajo. Dado que el capitalismo se basa 
en la mercantilización de la fuerza de trabajo y, teniendo en cuenta que la comida es uno de los componentes esenciales de su valor de cambio (expresado en el salario), siempre ha sido relevante mantener bajos los precios de los alimentos. Cuanto más bajos son los precios de los alimentos, menor es la presión que enfrentan los capitalistas por parte de los trabajadores para aumentar los salarios y mayor es la plusvalía obtenida que hace posible ampliar las ganancias. Por lo tanto, el capitalismo se convirtió en equivalente a la producción de alimentos baratos (Moore, 2015a), muchos de los cuales se producen bajo condiciones no capitalistas. En efecto: los alimentos han atravesado diversos modos de producción para alimentar a los trabajadores asalariados y varias naciones en la economía mundial. La forma en la que los alimentos han sido elaborados y comercializados ha dependido en gran medida de qué nación ha dominado la economía mundial en los diferentes momentos de su historia.

El marco analítico del régimen agroalimentario ha sido muy útil para entender la relación entre la agricultura y la acumulación de capital a escala mundial. De acuerdo con las conceptualizaciones de Harriet Friedmann y Philip McMichael (Friedmann \& McMichael, 1989; McMichael 2009, 2013), un régimen alimentario se refiere a una dinámica temporal específica de integración a la economía política agroalimentaria global. Se caracteriza por interacciones y relaciones que dan forma y a su vez son formadas por estructuras institucionales y reglas escritas y no escritas respecto de la agricultura y la alimentación que, a pesar de sus aspiraciones globales, son geográfica e históricamente específicas.

Tales dinámicas se combinan para crear un «régimen» cualitativamente distinto de las tendencias de acumulación de capital en la agricultura y la alimentación, cuya durabilidad se encuentra en la vinculación internacional de producción y consumo alimentarios, según las tendencias globales de acumulación de capital en términos más generales. $\mathrm{Ca}$ da régimen alimentario se basa, por lo tanto, en relaciones comerciales 
internacionales relativamente estables, aunque típicamente desiguales. Friedmann y McMichael identificaron con claridad dos regímenes alimentarios desde fines del siglo XIX: extensivo-colonizador, dominado por el imperio británico hasta la Primera Guerra Mundial; e intensivo-excedentario, que surgió después del periodo de transición tras la Segunda Guerra Mundial, dominado por Estados Unidos. Desde su artículo seminal de 1989, Friedmann y McMichael sugirieron que emergía un tercer régimen, al que McMichael denominó en trabajos posteriores «empresarial» (corporate). Por razones teóricas y políticas discutidas en otros textos (Pechlaner \& Otero, 2008, 2010; Otero, 2014), hemos preferido designarlo como régimen alimentario neoliberal, mismo que ha estado en operación a partir de la década de 1980 y que también ha sido dominado por Estados Unidos (Otero, Pechlaner y Gürcan, 2013).

El régimen alimentario neoliberal tiene como contrapartida la dieta neoliberal (Otero, Pechlaner, Liberman y Gürcan, 2015) compuesta por otras diferenciadas según la clase social. Por un lado, hay un predominio de los alimentos procesados, ricos en energía, desarrollados en un origen en Estados Unidos para sus clases trabajadoras - lo que se ha dado en llamar la «dieta industrial» (Winson, 2013) o la «dieta occidental» (Pollan, 2008). Desde nuestra perspectiva, la dieta neoliberal consiste en la globalización de la dieta industrial estadounidense. Los alimentos densos en energía son los más accesibles para la mayoría de la gente, en concreto para las clases de bajos y medianos ingresos. En el extremo opuesto del espectro de la dieta neoliberal referente a costos y precios, se encuentran los alimentos de lujo: varias carnes (Lang, 2010; Weis, 2013), frutas, verduras y otros productos de valor agregado, como el vino. El acceso a esos alimentos se limita a las clases de ingresos medio-altos y altos.

Gran parte de la dieta neoliberal se remonta a los cultivos transgénicos, es decir, los productos de la ingeniería genética (Kloppenburg, 2004), como el maíz y la soja que en Estados Unidos concentran la mayoría de 
los subsidios a la agricultura (Pollan, 2008: 117). Irónicamente, muchos de esos cultivos no se producen para el consumo humano directo. Se los utiliza más bien para la producción de ganado o alimentos procesados, incluyendo aceites vegetales y jarabe de maíz de alta fructosa. En el caso de la soja, sólo 6 por ciento de la producción mundial se consume en forma de granos, tofu u otro uso fermentado. El restante 94 por ciento se transforma en harina de soja y aceite que se destina a nuevas etapas de producción (Oliviera \& Schneider, 2016: 168).

No ha sido marginal el Estado en la conformación de la producción agroalimentaria. Nuestro concepto de «neorregulación» toma en cuenta el cambio de naturaleza de la intervención en el régimen alimentario neoliberal (Otero, 2014). Esta perspectiva sobre el Estado se contrapone a la concepción en boga, la cual minimiza su papel al usar el término «desregulación». Sólo con una adecuada concepción es factible concebir la esperanza de transformar la intervención estatal en una dirección más sostenible. El matiz sobre el Estado se pierde, por ejemplo, en la conceptuación que hace McMichael (2009) del régimen alimentario empresarial, pues no aparecen mediaciones entre la economía mundial o sistema-mundo y la dominación de las agroempresas multinacionales (AEM). Los artículos de este simposio resaltan dicho papel en cada estudio de caso y determinan la pertinencia del concepto de neorregulación.

Hemos propuesto que las tres diferencias fundamentales entre el segundo y el tercer régimen alimentario son las siguientes: 1) la transformación de la agricultura, de un enfoque nacional a uno global a través del comercio mundial; 2) la biotecnología se convierte en el principal motor de la producción, hecho que preserva la lógica de la Revolución Verde en tanto modernizadora de la agricultura y completa lo que Raj Patel (2013) ha nombrado «la larga Revolución Verde»; 3) el Estado cambia sus formas de intervención: de apoyar a la agricultura nacional y promover las AEM como agentes económicos primordiales. En esencia, el modelo 
neoliberal se basa en la integración económica mundial facilitada por los gobiernos a través de la neorregulación. El grado de integración ha sido desigual y combinado, debido en gran parte a los niveles diferenciales de desarrollo de las naciones y como resultado de distintas políticas estatales perseguidas por varios países.

La idea de «desregulación» de mercados y empresas asume la existencia de una importancia decreciente del Estado. El concepto de neorregulación, por el contrario, permite apreciar las nuevas formas de intervención de los Estados que promueven proyectos de desarrollo neoliberales, a la vez que también hace posible cuestionar estas políticas a nivel del Estado nación. Las políticas fundamentales del Estado han incluido las regulaciones nacionales y las internacionales. Entre otros aspectos, éstas últimas se han orientado a la homogeneización de los derechos de propiedad intelectual en diferentes Estados. Las políticas neoliberales se han promovido en primer lugar a través de organizaciones supraestatales como la Ronda de Uruguay del Acuerdo General sobre Aranceles y Comercio (GATT), que en 1995 se convirtió en la Organización Mundial de Comercio (OMC); y luego, gradualmente, a través de los acuerdos comerciales regionales como el Tratado de Libre Comercio de América del Norte (TLCAN) y otros tratados regionales. Tales acuerdos de libre comercio promovieron la homogeneización de los derechos de propiedad intelectual, que constituyen una ventaja competitiva central para las AEM y una libertad aún mayor en lo concerniente al movimiento de capitales privados, en especial de las AEM.

Mientras que la teoría del sistema-mundo inspiró en gran medida la perspectiva del régimen alimentario, argumentamos aquí que es necesario superar algunas de sus limitaciones, en específico la idea de que el sistema-mundo debe ser la unidad de análisis en cuanto escala y nivel privilegiado de abstracción en el que debe producirse el análisis (véase Arrighi $\&$ Moore, 2001). Dicho nivel de abstracción o de análisis nos confina a 
mirar al capital como un todo, en general, sin desagregar apropiadamente las diferentes fracciones del capital, junto con las probables contradicciones entre ellas. Tampoco conduce a un examen adecuado del Estado en la escala nacional. El sistema-mundo es, sin duda, un punto de partida o de llegada útil, pero es indispensable considerar otras mediaciones si queremos comprender con claridad cómo funciona la agencia social colectiva a escala del Estado nación y subnacional (las dos escalas en las cuales la mayoría de los movimientos sociales y organizaciones de la sociedad civil puede desplegar sus acciones). Sin tener en cuenta tales consideraciones es prácticamente imposible ir más allá de una mirada «desde arriba», en la que el capital se transforma en el demiurgo de todos los procesos sociales. Desde ese punto de vista, incluso el Estado se convierte en poco más que un epifenómeno del capital al actuar en su nombre.

Nuestra propuesta es que necesitamos una comprensión mucho más matizada del Estado que englobe la lucha de clases a diferentes escalas, desde el nivel nacional al subnacional. El Estado no sólo es relativamente autónomo respecto de las diversas fracciones del capital (Poulantzas, 1973; Jessop, 2007), sino que puede actuar a favor de las fuerzas populares-democráticas cuando existe suficiente presión «desde abajo». Lo que se precisa, por lo tanto, es un marco analítico que sea sensible a diferentes niveles de abstracción y prácticas, y que incorpore al análisis las distintas escalas, desde la localidad a la región, desde la nación a la economía mundial. Para lograrlo, Gerardo Otero (2004) propuso el enfoque de «vinculaciones desde abajo» o Vida.

El enfoque de Vida es metodológico y político. Es metodológico porque sugiere centrar el estudio en la vida real de las personas, las relaciones de explotación y opresión en las que participan, y sus peleas para convertirse en grupos y clases organizadas que pugnan por sus intereses. Además, si se requieren alianzas más amplias y solidaridad internacional funciona el enfoque desde abajo: a partir de las formas concretas en que las personas 
articulan sus intereses desde sus microecologías y vínculos determinados. En lo político, el enfoque de Vida prescribe que las organizaciones de escala más alta responden de manera subordinada a quienes se encuentran «abajo» o, como propone el dicho zapatista, «mandar obedeciendo». Considera al sistema-mundo como el objetivo último de transformación, pero es realista en lo referente a qué niveles pueden ser cambiados en el corto y mediano plazo (pese a que el cambio gradual pudiese ser visto como meramente reformista), al tiempo que trabaja en los intersticios del Estado y la sociedad hacia un proyecto popular-democrático emancipador.

Sin embargo, el Estado también puede contribuir a la expansión de las relaciones capitalistas, la explotación y el despojo, incluso cuando se trata de incorporar las demandas populares, como se ejemplifica en el caso de Argentina. Debemos, pues, ser conscientes del peligro inminente y siempre presente de la cooptación de grupos y clases subordinados. En ese sentido, la pregunta es: ¿̇en qué medida las organizaciones pueden extraer concesiones del Estado sin comprometer la autonomía para así poder continuar con la lucha de largo plazo en pos de sus intereses estratégicos? No abordaremos esa compleja cuestión en el presente artículo o en los que le siguen (Otero, 2006a; 2006b), si bien es importante valorar este tema clave en el enfoque de Vida.

\section{Acumulación por desposesión y valor negativo}

Primero establecemos la asociación conceptual entre acumulación originaria y acumulación por desposesión. Luego presentamos la idea de valor negativo como un nuevo concepto que puede contribuir al análisis de los cultivos transgénicos y el neoliberalismo en América Latina.

En su análisis de la acumulación originaria, Marx prescinde explícitamente «de los resortes puramente económicos de la revolución agrícola. 
De lo que nos ocupamos es de los medios violentos empleados por la misma» (1975: 904). Hace después un recuento histórico de las variadas formas que se agenció el capital para expropiar la tierra e introducirse en la agricultura, desde «la expoliación de los bienes eclesiásticos» y la «enajenación fraudulenta de las tierras fiscales» (es decir, del Estado o de la nación), hasta «el robo de la propiedad comunal» y «el terrorismo más despiadado» (Marx, 1975: 917). En síntesis, de acuerdo con Marx, si el dinero viene al mundo con manchas de sangre en una mejilla, «el capital lo hace chorreando sangre y lodo, por todos los poros, desde la cabeza a los pies» (1975: 950).

Con esas palabras dolorosamente poéticas, Marx refiere el proceso que creó las condiciones para que emergiera en Europa el modo de producción capitalista: la acumulación originaria y la creación de una fuerza de trabajo que se pudiera comprar y vender a cambio de un salario. Es este un proceso por el cual los productores directos se enfrentaron a una «doble libertad»: 1) fueron «liberados» de sus medios de subsistencia: la tierra, los medios de producción y las garantías feudales; y 2) quedaron en libertad de todas las relaciones de dependencia, tales como la servidumbre y los gremios del sistema feudal. Con la doble libertad, los productores directos se quedaron sin nada más que su fuerza de trabajo para intercambiar por un salario. Marx sitúa la acumulación originaria en «el último tercio del siglo XV y las primeras décadas del XVI» (1975: 894).

¿Qué tiene que ver este antiguo proceso, que se dio hace más de medio milenio, con la América Latina del siglo XXI? El hecho es que han sucedido varios procesos de expropiación en América Latina, en especial desde el inicio de la colonización europea en el siglo XVI. No obstante, desde la década de 1980, el vuelco neoliberal ha renovado el impulso de lo que los políticos llaman modernización y que, en una perspectiva crítica, puede caracterizarse como el establecimiento de condiciones que 
permiten una mayor penetración del capitalismo en la agricultura. Irónicamente, los gobiernos elegidos en la década de 2000, que se definen de izquierda o centro-izquierda y se comprometen con políticas «posneoliberales», siguen promoviendo en mayor o menor medida la modernización agrícola y la biotecnología.

En el proceso europeo, la acumulación originaria tuvo como contrapartida los inicios de la industrialización y la urbanización, por lo que muchos productores directos encontraron empleo asalariado. Un proceso similar ocurrió siglos después en Estados Unidos, donde los agricultores familiares fueron desplazados por la agricultura capitalista o subordinados a la agricultura moderna por los productores de insumos y la industria procesadora y distribuidora de alimentos. Uno de los dilemas perennes en América Latina es que la modernización agrícola no ha tenido como contrapartida un vigoroso proceso de industrialización que haya absorbido la población redundante del campo (Kay, 2008). Muchos de los que fueron contratados en el proceso de industrialización por sustitución de importaciones de los años 1930 a 1960 fueron eventualmente «ajustados» durante el vuelco neoliberal de la década de 1980 (Portes \& Hoffman, 2003). En resumen, en América Latina no se ha dado algún círculo virtuoso entre la expropiación en la agricultura y el empleo remunerado en las ciudades.

Sin embargo, la agricultura moderna ha cobrado impulso en América Latina con el inicio de la revolución biotecnológica a partir de la década de 1990. Una cuestión pendiente es si este proceso representa de modo simple una forma renovada de expropiación o si hay alguna contrapartida que mitigue la «doble libertad» que enfrentan los productores directos en la acumulación originaria o, como la denomina David Harvey (2003) en la época contemporánea, la acumulación por desposesión. Otra cuestión central se refiere a los impactos ecológicos de la revolución biotecnológica para las personas, las comunidades y la biodiversidad. 
$\mathrm{El}$ «valor negativo» es un nuevo concepto propuesto por Jason W. Moore (2015a; 2015b) con la intención de argumentar que la catástrofe ecológica causada por el capitalismo en el siglo XXI está haciendo mella en el propio proceso de capitalización. Alude a la combinación feroz de los crecientes costos de producción y el cambio climático. La idea de valor negativo conforma así una contradicción interna y potencialmente devastadora del capitalismo (Moore, 2015a: 5). Los artículos del simposio no aluden de forma directa o explícita a este fenómeno. Pero creemos que es relevante para entender el proceso de modernización de la agricultura en América Latina, como se muestra a continuación.

Desde sus inicios, la acumulación capitalista ha consistido en dos procesos, los cuales implican la apropiación del trabajo libre: uno es la capitalización, que entraña la apropiación de la plusvalía y el pago de salarios para cubrir los costos de reproducción de la fuerza de trabajo; es la esfera del conflicto trabajo-capital. El otro movimiento o proceso es lo que Moore nombra apropiación. También desde el comienzo del capitalismo sus propietarios se han adueñado de los recursos naturales y de partes de lo que corresponde al costo de reproducción de la fuerza de trabajo existente; por ejemplo, cuando la producción campesina de subsistencia subvenciona el trabajo asalariado realizado por algunos miembros de la familia campesina. Este subsidio extraído de la economía campesina permite que el salario capitalista quede por debajo del costo real de la fuerza de trabajo. Otro ejemplo son los migrantes en un nuevo destino laboral: el lugar de destino no ha pagado nada correspondiente a la producción y reproducción de dicha fuerza de trabajo y, no obstante, sus capitalistas están en condiciones de explotarlo.

En el caso de los recursos naturales, desde la década de 1970 los críticos ambientalistas del capitalismo han hablado de «externalidades» que no son asumidas por las empresas capitalistas contaminantes. Ello resulta cierto, pero como su nombre lo indica, la contaminación y otras 
formas de degradación ambiental que son «externas» a la acumulación de capital, no necesariamente hacen mella en la búsqueda de lucro o en su capitalización; más bien la permiten. El valor negativo, por el contrario, se presenta cuando los factores «externos» ya no son solamente externos a la acumulación de capital.

Valor negativo, explica Moore, se puede entender como «la acumulación de límites biofísicos para el capital (...) que ahora están restringiendo la restauración de las Cuatro Gangas [que siempre ha buscado el capitalismo]: alimentación, mano de obra, energía y materias primas» (2015a: 22). El primer límite es que la agricultura capitalista moderna se ha vuelto cada vez más ineficiente en términos energéticos. Mientras que en la década de 1930 se requerían cerca de 2.5 calorías para producir 1 caloría de alimento, la relación ha aumentado desde entonces a 7.5:1 en la década de 1950, y a 10:1 en la década de 1970: «En el siglo XXI se necesitan 15-20 calorías para lograr una caloría de alimentos desde la granja hasta la mesa, y considerablemente más que esto para la fruta fresca de origen global» (Moore, 2015a: 19). El segundo límite es que la agricultura constituye la fuente principal de intoxicación, por delante de la industria. Peor aún, «se necesitan más y más herbicidas y fertilizantes para producir cada incremento en el crecimiento (decreciente) de la productividad» (Moore, 2015a: 24). La mayoría de los autores del simposio documenta la creciente intoxicación de la agricultura moderna en América Latina, así como la resistencia desde abajo.

\section{Cultivos transgénicos en América Latina}

El simposio ofrece una visión general de la situación real existente respecto de la biotecnología agrícola en América Latina. Se examinan los impactos de la soja y el maíz transgénicos que, junto con el algodón y la 
canola, representan la mayor parte de los transgénicos que se cultiva en el mundo. Nuestro análisis se basa en tres propuestas acerca del vínculo entre la biotecnología agrícola y el neoliberalismo. En primer lugar, buscamos evitar las trampas del (bio)determinismo tecnológico. Es decir, suponemos que no es posible separar los cultivos GM de su contexto social más amplio, en el que las grandes AEM son las desarrolladoras y promotoras dominantes de transgénicos, a menudo sobre la base de las instituciones de investigación públicas locales como las universidades. De ese modo, evitamos enfoques descontextualizados de la biotecnología agrícola: la idea de que la biotecnología es un inexorable «desarrollo de las fuerzas productivas», la visión esencialista según la cual los cultivos transgénicos estarían «jugando con la madre naturaleza» y la concepción simplista de la biotecnología agrícola como una simple herramienta, una «bala de plata» contra el hambre en el mundo (con frecuencia se imagina así en concepciones neomalthusianas, tan difundidas entre las AEM, organismos internacionales y fundaciones filantrópicas).

En segundo lugar, sostenemos que la relación entre neoliberalismo y biotecnología agrícola puede no ser lineal, pero de cualquier forma existe una serie de afinidades electivas entre ambas. La neorregulación implica un fortalecimiento de la legislación que protege los derechos de propiedad intelectual, lo cual es clave para las AEM. Puesto que los cultivos transgénicos son patentados y promovidos por las AEM biotecnológicas y fueron comercializados y adoptados durante el apogeo del neoliberalismo en América Latina, representan la expresión tecnológica más clara del régimen alimentario neoliberal (Otero, 2014).

Por último, en tercer lugar, tratamos de ir más allá de la idea del neoliberalismo como ideología que pertenece exclusivamente a los sectores dominantes. Con ese propósito exploramos sus alternativas de adaptación al observar cómo el neoliberalismo moviliza subjetividades (por ejemplo, sectores de clase media y agricultores medios) y cómo sus 
principales actores se expanden y posicionan en el contexto del vuelco «posneoliberal» en varios países latinoamericanos. Este fenómeno se ilustra en el estudio de los entusiastas adoptadores de los cultivos transgénicos, en combinación con historias agrarias específicas: en Argentina, por ejemplo, las AEM venden los insumos de la agricultura transgénica (semillas y agroquímicos), pero los cultivan empresas agroindustriales argentinas y medianos y grandes agricultores. Nuestro simposio se focaliza en esos tres puntos.

La mayoría de los autores del simposio utiliza el concepto de «acumulación por desposesión» de David Harvey a fin de describir los procesos de expropiación que tienen lugar en sus estudios de caso. En opinión de Harvey (2003), tal proceso está en curso en pleno siglo XXI, sobre todo en los llamados países en desarrollo: el capitalismo avanza en cualquier lugar donde existan opciones de lucro. La peculiaridad de la modernización agrícola, como siempre, es que no sólo se expropia a los productores directos, también se les despoja de su tierra al considerarla como cualquier otra actividad extractiva: se abusa de la fertilidad de la tierra, mientras que se utilizan agroquímicos con efectos contaminantes. Pese a lo anterior, los principales beneficiarios de ese modelo de desarrollo deben presentarlo en términos positivos con el interés de hacerlo aceptable.

Carla Gras y Valeria Hernández examinan la legitimación de la biotecnología agraria en Argentina y el papel que desempeñaron las clases capitalistas nacionales en el proceso. Su artículo ofrece un análisis en profundidad de la función de la tecnología no sólo como un mero instrumento de la producción, sino como una ideología promovida para legitimar una comprensión específica del desarrollo agrario, coherente con el mantenimiento del liderazgo económico e ideológico de la burguesía local agraria. La biotecnología fue presentada como la solución al estancamiento de la agricultura, extrayendo su componente político, es decir, la función social y la distribución de la tierra. Utilizando el 
concepto de «revolución pasiva» de Antonio Gramsci, la idea generalizada de que la biotecnología en Argentina ha dado lugar a una revolución agrícola es discutida por Gras y Hernández. Al contrario, vinculada al neoliberalismo y al paradigma del agronegocio, la biotecnología ha asegurado la continuidad económica e ideológica de la Revolución Verde. La adopción de la biotecnología por una multiplicidad de agricultores ha significado la aceptación del liderazgo de la burguesía agraria local.

Amalia Leguizamón documenta cómo la agricultura transgénica se inscribe en el renovado impulso del extractivismo de la década de 2000, en la que los gobiernos promueven la modernización y la colocan en manos de grandes AEM. La contrapartida del impulso a la acumulación por desposesión es lo que se ha denominado luchas contra el despojo (Cáceres, 2015). Leguizamón se centra en las luchas del movimiento campesino-indígena y de las organizaciones en contra de la fumigación con agroquímicos en Argentina.

Marla Torrado presenta un estudio de los gobiernos progresistas de los Kirchner en Argentina (2003-2015) y su apoyo a la modernización agrícola con el empleo de transgénicos, al tiempo que supuestamente implementaron un "posneoliberalismo». A través de un análisis documental Torrado muestra que, lejos de representar una ruptura con el neoliberalismo, tales gobiernos significaron una continuidad de la política de Estado en lo que se refiere a la agricultura. Los grandes actores económicos transnacionales que respaldan esta modernización también han promovido una forma de gobierno y de política tecnológica sin precedentes. Por lo tanto, la agenda posneoliberal fomenta la producción de cultivos transgénicos pese a los problemas ecológicos y los daños a la salud de las comunidades, a la vez que aumentan los programas de asistencia social.

Arturo Ezquerro Cañete evidencia cómo el régimen soyero neoliberal se ha extendido desde Argentina y Brasil hacia uno de los países más pequeños de América del Sur: Paraguay. Un país que exhibe uno de los 
más altos índices de pobreza ha sido impactado por lo que Ezquerro Cañete llama con acierto «acumulación por desposesión y fumigación». La modernización de la agricultura dio lugar a consecuencias nefastas para la subsistencia rural. Debido a las fumigaciones, numerosos campesinos fueron expulsados de sus comunidades y muchas de sus propiedades han acrecentado las fuentes para la acumulación del capital, lo que los deja sin ningún medio de subsistencia. Por tanto, es urgente encontrar un modelo agrario adecuado, sostenible y ético que absorba a dicha población.

La poderosa fuerza de la acumulación por desposesión ha encontrado resistencias en América Latina. De hecho, teniendo en cuenta cómo afecta profundamente la base material de reproducción social de grupos y clases subordinados, algunos movimientos vigorosos surgen desde abajo para defender a la sociedad en diversas escalas — subnacionales, nacionales y transnacionales-, como se muestra en los tres artículos siguientes.

Laura María Gutiérrez Escobar y Elizabeth Fitting presentan un análisis etnográfico de la Red de Semillas Libres en Colombia (RSL), que vincula a activistas y organizaciones de base. Mediante proyectos que relacionan las semillas con la soberanía, la desobediencia civil y las demandas legales, la RSL se abre paso con su lucha para rechazar los transgénicos y proteger las variedades de plantas tradicionales, los conocimientos y las prácticas ligados a las semillas. La RSL desafía aspectos clave del régimen alimentario neoliberal en Colombia, en particular los nuevos derechos de propiedad intelectual y las normas sobre semillas adoptadas como parte del acuerdo del libre comercio con Estados Unidos. Basados en el concepto de biohegemonía, Gutiérrez y Fitting argumentan que la RSL cuestiona los supuestos del discurso y la política oficial acerca de las variedades de semillas tradicionales, a las que consideran como materias primas y recursos a ser «descubiertos», «inventados» o mercantilizados por la industria y la ciencia occidental. 
Renata Motta explora la dinámica política alrededor de los transgénicos en Brasil y destaca el papel de la neorregulación. Su artículo muestra que la política estratégica alrededor de los cultivos transgénicos se centra en el Estado nación, tanto para los grupos dominantes que los promueven como para los grupos subordinados que los cuestionan. Su estudio evidencia que el Estado nación sigue siendo central en la aplicación de un régimen agroalimentario sustentado en la biotecnología y en las luchas en su contra. Siguiendo a Saturnino Borras Jr., Motta argumenta en contra de los estudios dominantes que despolitizan la investigación y las políticas públicas a través de conceptos como «gobernabilidad», o al equiparar la política a los fenómenos negativos de la corrupción o los Estados fallidos. Este tipo de investigaciones legitima la desaparición del desarrollo dirigido por el Estado para favorecer las políticas orientadas al mercado. Motta propone tomar en serio a la política e involucrar a las relaciones Estado-sociedad en la dinámica del cambio agrario. También ilustra este punto al examinar cómo los actores subordinados resisten a los cultivos GM en Brasil e influyen en la política oficial a nivel del Estado nación.

Por último, Irma Gómez González ofrece una nueva exploración de los efectos ecológicos desastrosos que causó la autorización de la soja transgénica en la península de Yucatán, México, en los bosques locales, lo que impactó profundamente la economía de los apicultores mayas. La apicultura había llegado a ser la principal fuente de subsistencia para la mayoría de los campesinos mayas en Campeche. Después de su autorización por parte de los gobiernos federal y estatal en 2011, la soja transgénica representaba una amenaza para esa economía. Al tiempo que se autorizaba la soja transgénica, la Unión Europea anunció un nuevo requisito para la miel importada: que debería ser etiquetada en el caso de contener transgénicos. Los importadores de miel requirieron así una etiqueta que indicara si la miel era «libre de transgénicos». El artículo 
de Gómez González documenta la lucha popular en contra de la soja transgénica, una amplia alianza que incluye comunidades mayas, apicultores, organizaciones de la sociedad civil, universidades y empresarios exportadores de miel. Utilizando ingeniosas estrategias mediáticas, esta alianza finalmente consiguió una victoria en el poder judicial mexicano al lograr invalidar la autorización de la producción de soja transgénica. El caso ilustra el modo en que el valor negativo desafía a la capitalización en ciertos sectores de la economía capitalista. La convergencia de políticas nacionales y extranacionales presenta oportunidades específicas para la movilización que pueden conducir a un exitoso cambio de política a nivel del Estado nación.

En suma, este simposio expone análisis históricos concretos de la manera en que ha procedido la acumulación de capital en la agricultura en la era del neoliberalismo, incluyendo el estudio de sus fuerzas dinámicas, las consecuencias y posibles vías de cambio por medio de movilización y vinculaciones desde abajo (Vida). Inicialmente sugerimos la posibilidad de que los distintos casos de América del Sur representaran el mayor avance de la agricultura moderna, con el liderazgo de AEM y poderosas burguesías agrarias locales. ¿En qué medida esto se puede convertir en el patrón dominante de la región? ¿Se está consolidando la concentración gracias a las poderosas fuerzas combinadas de las políticas neoliberales y la tecnología transgénica? ¿Es el desafío de la resistencia y vinculaciones desde abajo lo suficientemente fuerte como para modificar el curso de los acontecimientos hacia una dirección popular-democrática? Tales interrogantes quedan pendientes para futuras investigaciones, las cuales deben responderse de acuerdo con las especificidades históricas.

Al emplear la metodología de Vida, «el capital» en general sería visto como una abstracción a estudiar en sus manifestaciones concretas, no sólo para entenderlo, sino para resistirlo y desafiarlo con un enfoque de vinculaciones de abajo. $\mathrm{Al}$ respecto, no se rechaza el globalismo 
cosmopolita, siempre y cuando responda y rinda cuentas a las organizaciones de base y modifique su enfoque neoliberal. Puesto que el Estado sigue desempeñando un papel destacado en el régimen alimentario neoliberal, también debe ser un objeto de lucha para grupos y clases subordinados. Los medios de vida y la supervivencia de las ecologías locales se encuentran en peligro, por lo que surgen nuevas y múltiples alianzas de actores diversos en los intersticios de la sociedad civil. La transformación social emancipadora depende de que las fuerzas populares-democráticas puedan aprovechar los intersticios emergentes con fuertes vinculaciones desde abajo.

\section{Referencias}

Arrighi, G. \& Moore, J.W. (2001). «Capitalist Development in World Historical Perspective». En Albritton, R., Itoh, M. \& Westra, R. (eds.), Phases of Capitalist Development: Booms, Crises and Globalization. New York: Palgrave, pp. 56-75.

Cáceres, D.M. (2015). «Accumulation by Dispossession and Socio-Environmental Conflicts Caused by the Expansion of Agribusiness in Argentina». Journal of Agrarian Change 15(1), pp. 116-147.

Fitting, E. (2011). The Struggle for Maize: Campesinos, Workers, and Transgenic Corn in the Mexican Countryside. Durham: Duke University Press.

Friedmann, H. \& McMichael, P. (1989). «Agriculture and the State System: The Rise and Decline of National Agricultures, 1870 to the Present». Sociologia Ruralis 29(2), pp. 93-117.

Harvey, D. (2003). The New Imperialism. Oxford: Oxford University Press. Hetherington, K. (2013). «Beans before the Law: Knowledge Practices, Responsibility, and the Paraguayan Soy Boom». Cultural Anthropology 28(1), pp. $65-85$. 
Hisano, S. \& Altoé, S. (2008). «Brazilian Farmers at a Crossroads: Biotech Industrialization of Agriculture or New Alternatives for Family Farmers». En Otero, G. (ed.), Food for the Few: Neoliberal Globalism and Biotechnology in Latin America. Austin: University of Texas Press, pp. 243-265.

Hui, A. (enero 13, 2016). «P is for Pulses». The Globe and Mail, pp. A6-A7. James, C. (2012), «Global Status of Commercialized Transgenic Crops: 2012». ISAAA Briefs 44, pp. 1-24.

Jessop, B. (2007). State Power: A Strategic-Relational Approach. Malden: Polity Press.

Kay, C. (2008). «Reflections on Latin American Rural Studies in the Neoliberal Globalization Period: A New Rurality?» Development and Change 39(6), pp. 915-943.

Klepek, J. (2012). «Against the Grain: Knowledge Alliances and Resistance to Agricultural Biotechnology in Guatemala». Canadian Journal of Development Studies 33(3), pp. 310-325.

Kloppenburg, J.R. (2004). First the Seed: The Political Economy of Plant Biotechnology (2nd ed.). Wisconsin: Madison University of Wisconsin Press.

Lang, T. (2010). «Crisis? What Crisis? The Normality of the Current Food Crisis». Journal of Agrarian Change 10(1), pp. 87-97.

Lapegna, P. (2013). «The Expansion of Transgenic Soybeans and the Killing of Indigenous Peasants in Argentina». Societies without Borders: Human Rights and the Social Sciences 8(2), pp. 291-308.

Leguizamón, A. (2014). «Modifying Argentina: GM Soy and Socio-Environmental Change». Geoforum 53, pp. 149-160.

McMichael, P. (2009). «A Food Regime Genealogy». Journal of Peasant Studies 36(1), pp. 139-169.

McMichael, P. (2013). Food Regimes and Agrarian Questions. Halifax: Fernwood. Marx, K. (1975). El capital (tomo I, vol. 3). México: Siglo XXI Editores.

Moore, J.W. (2015a). «Cheap Food and Bad Climate: From Surplus Value to Negative Value in the Capitalist World-Ecology». Critical Historical Studies 2(1), pp. 1-43. 
Moore, J.W. (2015b). Capitalism in the Web of Life: Ecology and the Accumulation of Capital. New York: Verso.

Motta, R. (2016). Social Mobilization, Global Capitalism and Struggles over Food: A Comparative Study of Social Movements. New York: Routledge.

Oliveira, G. de L.T. \& Schneider, M. (2016). «The Politics of Flexing Soybeans: China, Brazil and Global Agroindustrial Restructuring». Journal of Peasant Studies 43(1), pp. 167-194.

Otero, G. (2004). «Global Economy, Local Politics: Indigenous Struggles, Civil Society and Democracy». Canadian Journal of Political Science 37(2), pp. 325-346.

Otero, G. (2006a). «Forjando democracia: formación político-cultural y vinculaciones desde abajo». Iconos. Revista de Ciencias Sociales 26, pp. 131-146.

Otero, G. (2006b). México en transición: globalismo neoliberal, Estado y sociedad civil. México: Miguel Ángel Porrúa.

Otero, G. (2014). La dieta neoliberal: globalización y biotecnología agricola en las Américas. México: Miguel Ángel Porrúa.

Otero, G., Pechlaner, G. \& Gürcan, E.C. (2013). «The Political Economy of 〈Food Security»: Uneven and Combined Dependency». Rural Sociology 78(3), pp. 263-289.

Otero, G., Pechlaner, G., Liberman, G. \& Gürcan, E.C. (2015). «The Neoliberal Diet and Inequality in the United States». Social Science and Medicine 142, pp. 47-55.

Patel, R. (2013). «The Long Green Revolution». Journal of Peasant Studies 40(1), pp. 1-63.

Pechlaner, G. \& Otero, G. (2008). «The Third Food Regime: Neoliberal Globalism and Agricultural Biotechnology in North America». Sociologia Ruralis 48(4), pp. 351-371.

Pechlaner, G. \& Otero, G. (2010). «The Neoliberal Food Regime: Neoregulation and the New Division of Labor in North America». Rural Sociology 75(2), pp. 179-208. 
Pengue, W.A. (2005). «Transgenic Crops in Argentina: The Ecological and Social Debt». Bulletin of Science, Technology E Society 25(4), pp. 314-322. Pollan, M. (2008). In Defense of Food: An Eater's Manifesto. New York: Penguin. Portes, A. \& Hoffman, K. (2003). «Latin American Class Structures: Their Composition and Change during the Neoliberal Era». Latin American Research Review 38(1), pp. 41-82.

Poulantzas, N. (1973). Political Power and Social Classes, London: New Left Books.

Teubal, M. (2008). «Genetically Modified Soybeans and the Crisis of Argentina's Agriculture Model». In Otero, G. (ed.), Food for the Few: Neoliberal Globalism and Biotechnology in Latin America. Austin: University of Texas Press, pp. 189-216.

Weis, T. (2013). The Ecological Hoofprint: The Global Burden of Industrial Livestock. New York: Zed Books.

Winson, A. (2013). The Industrial Diet: The Degradation of Food and the Struggle for Healthy Eating. Vancouver: UBC Press. 